\title{
Global analysis of expression profile of members of DnaJ gene families involved in capsaicinoids synthesis in pepper (Capsicum annuum $\mathrm{L}$ )
}

Fang Fei Fan ${ }^{1 \dagger}$, Fawan Liu' ${ }^{2 \dagger}$, Xian Yang ${ }^{1}$, Hongjian Wan $^{3,4^{*}}$ (D) and Yunyan Kang ${ }^{1 *}$

\begin{abstract}
Background: The DnaJ proteins play critical roles in plant development and stress responses. Recently, seventy-six DnaJ genes were identified through a comprehensive bioinformatics analysis in the pepper genome. However, there were no reports on understanding of phylogenetic relationships and diverse expression profile of pepper DnaJ genes to date. Herein, we performed the systemic analysis of the phylogenetic relationships and expression profile of pepper DnaJ genes in different tissues and in response to both abiotic stress and plant hormones.

Results: Phylogenetic analysis showed that all the pepper DnaJ genes were grouped into 7 sub-families (sub-family I, II, III, IV V V, VI and VII) according to sequence homology. The expression of pepper DnaJs in different tissues revealed that about 38\% (29/76) of pepper DnaJs were expressed in at least one tissue. The results demonstrate the potentially critical role of DnaJs in pepper growth and development. In addition, to gain insight into the expression difference of pepper DnaJ genes in placenta between pungent and non-pungent, their expression patterns were also analyzed using RNA-seq data and qRT-PCR. Comparison analysis revealed that eight genes presented distinct expression profiles in pungent and non-pungent pepper. The CaDnaJs co-expressed with genes involved in capsaicinoids synthesis during placenta development. What is more, our study exposed the fact that these eight DnaJ genes were probably regulated by stress (heat, drought and salt), and were also regulated by plant hormones (ABA, GA3, MeJA and SA).

Conclusions: In summary, these results showed that some DnaJ genes expressed in placenta may be involved in plant response to abiotic stress during biosynthesis of compounds related with pungency. The study provides wide insights to the expression profiles of pepper DanJ genes and contributes to our knowledge about the function of DnaJ genes in pepper.
\end{abstract}

Keywords: DnaJ protein, Pepper, Expression analysis, Phylogenetics, Abiotic stresses

\footnotetext{
* Correspondence: wanhongjian@sina.com; kangyunyan@scau.edu.cn

${ }^{\dagger}$ Fang Fei Fan and Fawan Liu contributed equally to this work.

${ }^{3}$ State Key Laboratory for Managing Biotic and Chemical Threats to the

Quality and Safety of Agro-products, Institute of Vegetables, Zhejiang

Academy of Agricultural Sciences, Hangzhou 310021, PR China

${ }^{1}$ College of Horticulture, South China Agricultural University, Guangzhou

510642, PR China

Full list of author information is available at the end of the article
}

(c) The Author(s). 2020 Open Access This article is licensed under a Creative Commons Attribution 4.0 International License, which permits use, sharing, adaptation, distribution and reproduction in any medium or format, as long as you give appropriate credit to the original author(s) and the source, provide a link to the Creative Commons licence, and indicate if changes were made. The images or other third party material in this article are included in the article's Creative Commons licence, unless indicated otherwise in a credit line to the material. If material is not included in the article's Creative Commons licence and your intended use is not permitted by statutory regulation or exceeds the permitted use, you will need to obtain permission directly from the copyright holder. To view a copy of this licence, visit http://creativecommons.org/licenses/by/4.0/ The Creative Commons Public Domain Dedication waiver (http://creativecommons.org/publicdomain/zero/1.0/) applies to the data made available in this article, unless otherwise stated in a credit line to the data. 


\section{Background}

The DnaJ protein was originally identified in Escherichia coli as a $41-\mathrm{kDa}$ heat shock protein [1]. Generally, it was composed of four domains, including a J-domain, a G/Fdomain, a zinc finger (CxxCxGxG) domain, and Cterminal sequences [2-5]. Previous research has attempted to separate DnaJ protein into three groups (I/ II/III) according to the characteristics of structure [6]. Group I consisted of the J-domain, G/F-domain, and zinc finger domain. Group II was composed of the J-domain and either a G/F- or a zinc finger- domain. Group III have only the J-domain [7].

Plant growth and development is a complex biological process regulated by a network of various mechanisms. Numerous DnaJ proteins have been reported to participate and play important role in this process. It is well known that photosynthesis which takes place in chloroplast is a vital physiological process to plant growth and development [8]. Previously, researchers have revealed that DnaJ proteins were significant for chloroplast development. For instance, the DnaJ protein ARC6 located in chloroplast membrane of Arabidopsis was functions as a key factor essential for chloroplast differentiation by assembling or stabling the FtsZ rings [9]. Subsequently, Chen et al. [10] found that both specific and cross-talk functions exist in the three small chloroplast-targeted DnaJ proteins, AtJ8, AtJ11 and AtJ20. These small proteins participate in multiple physiological and biochemical processes, including optimization of $\mathrm{CO}_{2}$ fixation, in stabilization of PSII complexes and balancing the electron transfer reactions [10]. Recently, members of the DnaJ-like zinc finger domain proteins in Arabidopsis thaliana have been reported to participate in the biogenesis and/or the maintenance of plastids. One of the protein family members, PSA2, was found to localize to the thylakoid lumen and regulate the accumulation of photosystem I. The result demonstrated that PSA2 affects chloroplast development [11]. What's more, considerable research about the function of the DnaJ protein in rice was consistent with that reported previously. Zhu et al. [12] clearly illuminated that the OsDjA7/8 is required for chloroplast development in rice and it may act in relation with other proteins directly or indirectly.

The plant DnaJ functions were not only related to plant growth and development, but also were involved in plant resistance to abiotic stresses. The BIL2 gene localized in mitochondrial was classified as a member of the DnaJ family [13]. BIL2-overexpression plants showed resistance against salinity stress and strong light stress in Arabidopisis thaliana [14]. A chloroplast-targeted DnaJ protein (LeCDJ1) was isolated from tomato (Solanum lycopersicum). The expression was upregulated under chilling stress. Furthermore, the researchers further reported that overexpression of $L e C D J 1$ improved tolerance of PSII to low temperature stress, whereas inhibition of $L e C D J 1$ enhanced the sensitivity of PSII to low temperature. The result showed that LeCDJ1 played an important role in maintaining PSII under low temperature stress [15]. In 2015, the second tomato chloroplast-targeted DnaJ protein, SlCDJ2, was identified in transgenic tomatoes. The researchers reported that, under heat stress, over-expression of SlCDJ2 exhibited higher Rubisco activity, Rubisco large subunit content, and $\mathrm{CO}_{2}$ assimilation capacity but lower in antisense plants compared with wild-type plants. The results indicated that overexpression of SlCDJ2 in tomato improved its thermotolerance by alleviating heat stress-induced damage of Rubisco and facilitated, whereas its inhibition enhanced Rubisco damage and reducing heat-induced damage.

Pepper (Capsicum annum L.), one of important vegetable crops, had been widely cultivated around the world [16]. The pungency of chili fruits, one of the most important ingredients, is due to a group of chemical analogs known as capsaicinoids [17]. Although the biosynthetic pathways (the phenylpropanoid pathway and the branched-chain fatty acid pathway) of capsaicinoid have been reported [18], the factors that regulate these pathways are not clear.

In recent years, the sequencing of whole plant genomes has provided an opportunity to identify different gene families [19-22]. In previous study, we identified a total of 76 putative pepper DnaJ genes (CaDna)01 to CaDnaJ76) using bioinformatics methods in the pepper genome [23]. A genome-wide analysis of CaDnaJ gene family was performed to reveal gene structure, conserved motifs, chromosomal localization, cis-element and expression profiles in different tissues (root, stem, leaf, and pericarp) and heat stress condition. A number of stressrelated cis-elements were found in the promoter region of most CaDnaJ genes, suggesting that the CaDnaJs might be involved in the response process of complex stress conditions [23].

In the current study, to shed light into their underlying relationships, two pepper breeding lines (007EA-pungent and P2-nonpungent) were selected for analyzing coexpression patterns of the factors related capsaicinoid biosynthesis and $\mathrm{CaDnaJ}$ genes. Moreover, these CaDnaJ genes response to multiple stresses were also performed using qRT-PCR. These results will facilitate the functional studies of pepper DnaJs in the future.

\section{Results}

Phylogenetic analysis of pepper CaDnaJ genes

Based on the sequence homology of the 76 DnaJ protein sequences, the pepper DnaJ gene family was grouped into 7 sub-families (sub-family I, II, III, IV, V, VI and 


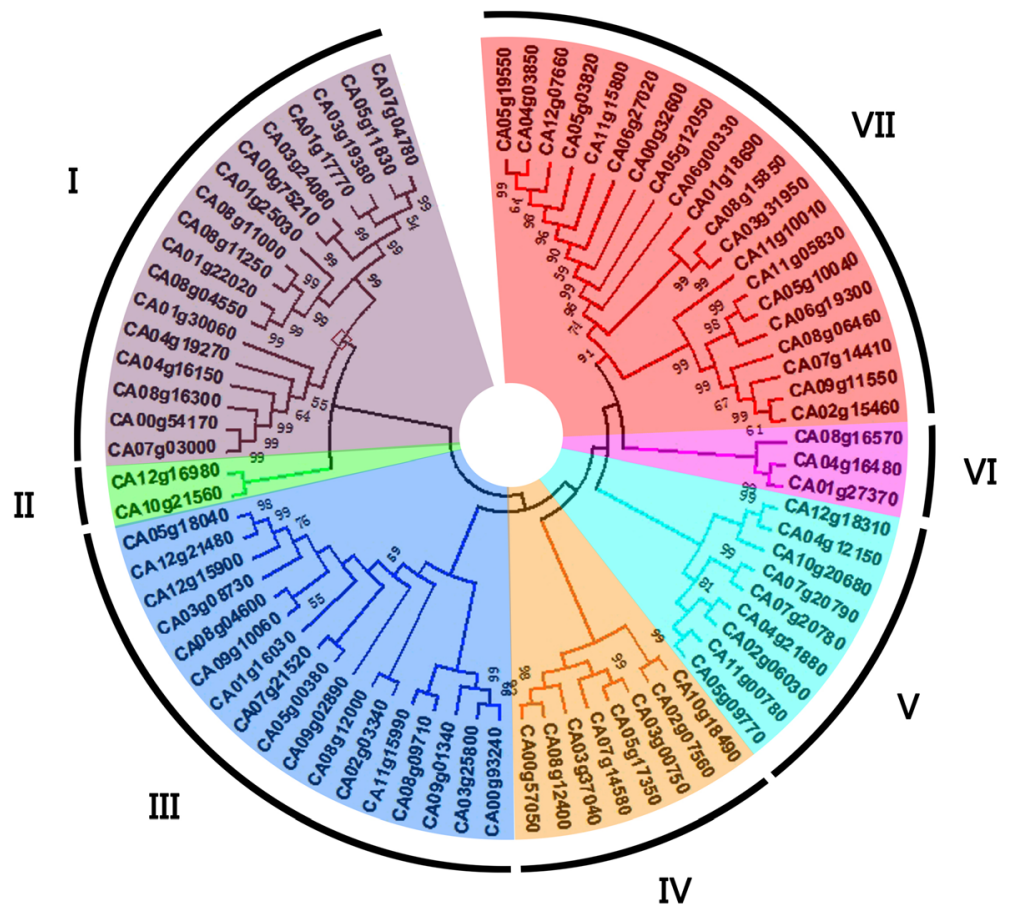

Fig. 1 Phylogenetic relationship of the identified 76 CaDnaJ genes in CM334 hot pepper. These genes were divided into seven subfamilies (I, II, III, IV, V, VI and VII)

VII). As shown in Fig. 1, Sub-family VII (containing 20 members) was the largest group, followed by sub-family I (17), sub-family III (17), sub-family V (9), sub-family IV (8), and sub-family VI (3). The sub-family II was the smallest, with only two CaDnaJ gene members (Fig. 2).

\section{Expression profiles of pepper CaDnaJ genes}

Plant tissue/organ-specific expression analysis based on RAN-seq and qRT-PCR technology can provide vital clues about the function differentiation of the genes in different tissue/organs [24-27]. To compare in detail the

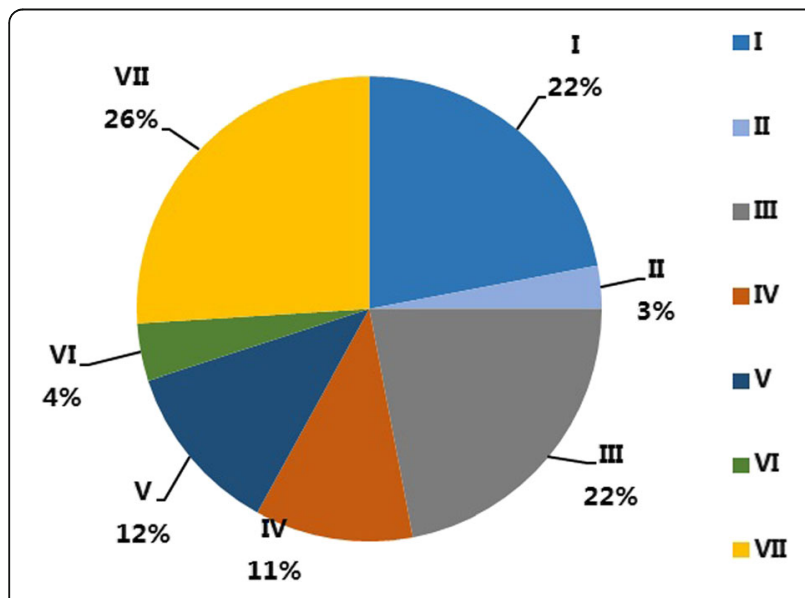

Fig. 2 Subfamily constitution of the identified 76 CaDnaJs in CM334
CaDnaJ expression in CM334, the RNA-seq data of the $\mathrm{CaDnaJ}$ genes were selected for further analysis. Different tissues from CM334, including root, stem, leaf, seven stages of pericarp and placenta (6 DPA, 16 DPA, 25 DPA, MG, B, B5, B10, respectively), were selected for expression analysis in the present study.

We found that 29 out of identified $76 \mathrm{CaDnaJ}$ genes were expressed in at least one tissue ([RPKM] $\geq 5.0$ was defined as expressed) (Supplement Table 2). The analysis on the constitutions of each subfamily revealed the differential expressions of CaDnaJ subfamilies in tested tissues (Table 1). The subfamily III members possess over $34 \%$ of the expressed CaDnaJ in tested tissues (Fig. 3), although it possesses only $22 \%$ of the total detected CaDnaJs (Fig. 2), indicating a higher expression

Table 1 Subfamily constitution of expressed CaDnaJ genes in different tissues in pepper

\begin{tabular}{lllllllllll}
\hline & Leaf & $\%$ & Root & $\%$ & Stem & $\%$ & Pericarp & $\%$ & Placenta & $\%$ \\
\hline I & 1 & 0.07 & 5 & 0.2 & 3 & 0.17 & 6 & 0.24 & 5 & 0.21 \\
III & 8 & 0.53 & 10 & 0.42 & 8 & 0.44 & 9 & 0.36 & 9 & 0.37 \\
IV & 0 & 0 & 3 & 0.13 & 1 & 0.06 & 3 & 0.12 & 3 & 0.13 \\
V & 3 & 0.2 & 3 & 0.13 & 3 & 0.16 & 3 & 0.12 & 3 & 0.13 \\
VI & 1 & 0.07 & 1 & 0.04 & 1 & 0.06 & 1 & 0.04 & 1 & 0.04 \\
VII & 2 & 0.13 & 2 & 0.08 & 2 & 0.11 & 3 & 0.12 & 3 & 0.12 \\
Total & 15 & 100 & 24 & 100 & 18 & 100 & 25 & 100 & 24 & 100 \\
\hline
\end{tabular}




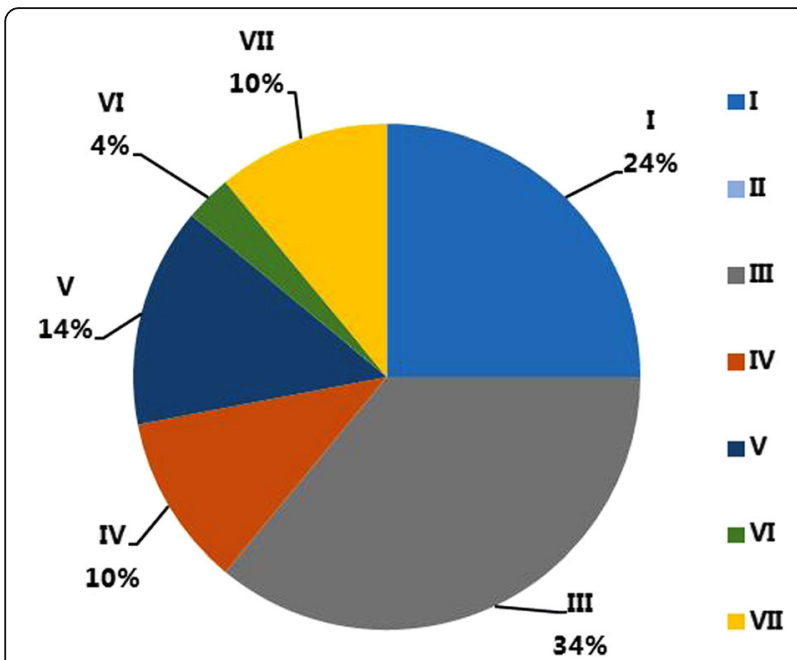

Fig. 3 CaDnaJ genes expressed in the investigated plant tissues based on transcriptome data in CM334. RPKM value over 5.0 is defined as expressed genes. 29 CaDnaJs are expressed in at least one tissue. Proportions of CaDnaJ subfamilies are showed in the figure

level and possible crucial roles in pepper development. In contrast, $26 \%$ of detected CaDnaJs belong to the subfamily VII (Fig. 2), while the expressed subfamily VII genes in the tested tissues possess only $10 \%$ in all analyzed tissues (Fig. 3). Interestingly, a special situation was found in subfamily II members, the two members were not expressed in the tested tissues (Fig. 3, Supplement Table S2).

\section{Expression patterns of CaDnaJ genes in various tissues}

Twenty-nine CaDnaJ genes in different tissues with RPKM value greater than 5.0 were selected for further analysis (Supplement Table S2). As it shown in Fig. 4, few CaDnaJs were specifically expressed in the tested tissues, and most CaDnaJs were expressed in two or more tissues. Data analysis showed that the number of specifically expressed CaDnaJs in vegetative tissue (leaf, root, and stem) was higher than that in reproductive tissue (pericarp and placenta) (Table 2). Additionally, more $\mathrm{CaDnaJ}$ genes were preferentially expressed in pericarp compared to the other tissues. Leaf and placenta share the same quantity of the preferentially expressed genes (Table 2).

\section{Expression patterns of CaDnaJ genes in placenta}

For transcriptome analyses of fruit, pericarp and placenta were harvested from CM334 (pungent) plants at the stages of 6 days post-anthesis (DPA), 16 DPA, 25 DPA, mature green (MG), breaker (B), 5 days postbreaker (B5), and B10. And in ECW30R (non-pungent) plants, placenta was harvested at 6 DPA, 13 DPA, 20 DPA, MG, B, B5, and B10 stages [16]. To reveal whether the expression of DnaJ is involved in the synthesis of capsaicin, CM334 and ECW30R were used to investigate expression patterns of CaDnaJ genes in placenta. Comparison analysis of RNA-seq data revealed that eight genes out of expressed 29 CaDnaJs (CaDnaJ25, 47 and 56 from sub-family III, CaDnaJ10, 40 and 74 from sub-family IV, CaDnaJ70 from sub-family $\mathrm{V}$ and CaDnaJ46 from sub-family VII) exhibited distinct expression profiles in CM334 and ECW (Supplemental Figure S1).

For CM334, the expression of CaDnaJ10, 25 and 40 increased gradually before the stage of MG and then remained high expression, while $\mathrm{CaDnaJ} 47$ and 70 remained stable during the development of placenta. In particular, the CaDnaJ46 was barely expressed before the stage of $\mathrm{B}$, and then dramatically increased in the later period. The expression of CaDnaJ56 were dramatically

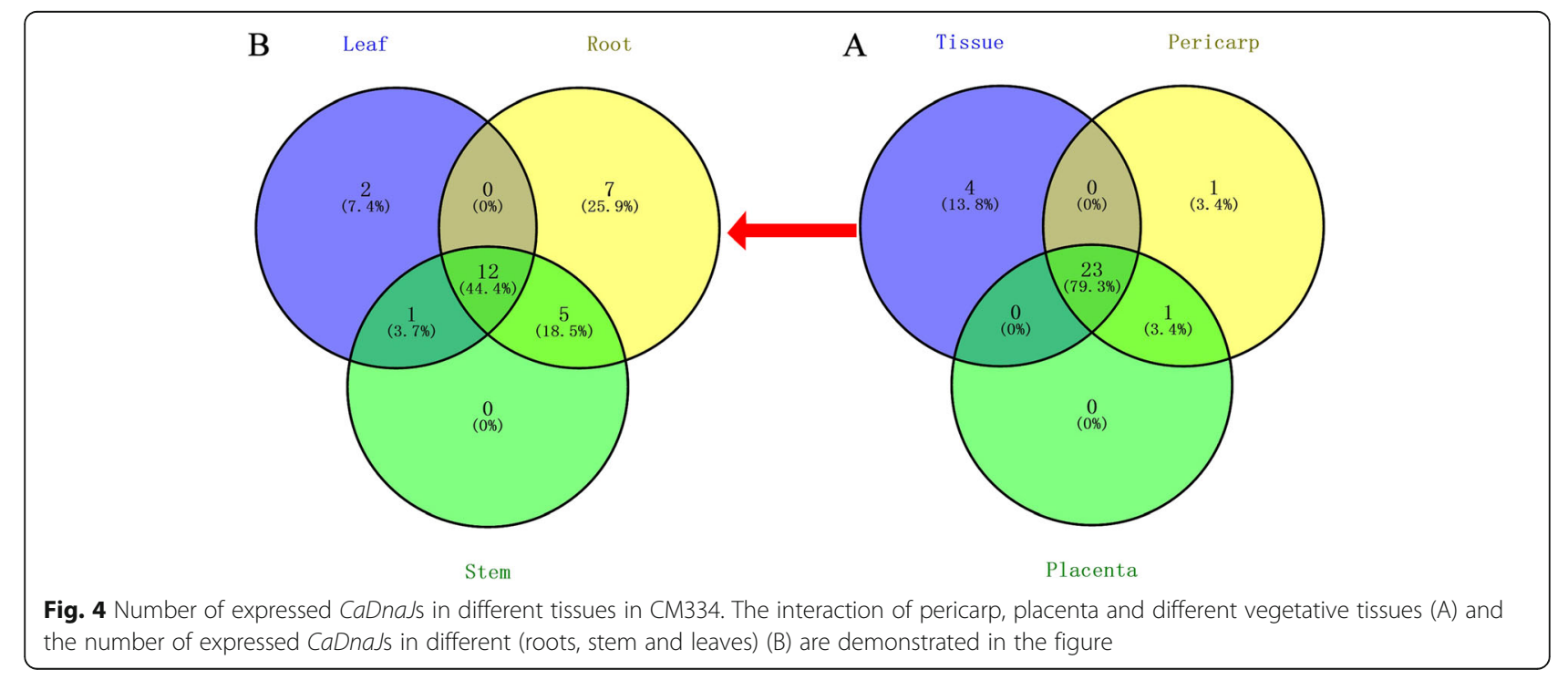


Table 2 The number of expressed, preferentially expressed, and specifically expressed CaDnaJ genes in different tissues in pepper

\begin{tabular}{llllll}
\hline Type & Leaf & Root & Stem & Pericarp & Placenta \\
\hline Expressed CaDnaJs & 15 & 24 & 18 & 25 & 24 \\
Preferentially expressed CaDnaJs & 5 & 6 & 3 & 10 & 5 \\
Specifically expressed CaDnaJs & 2 & 7 & 0 & 1 & 0
\end{tabular}

down-regulated in early stage and remained stable (Supplemental Figure S1).

In ECW, the expression of CaDnaJ25, 47 and 74 increased gradually at the early stage of placenta development, and then remain low expression. The expression of CaDnaJ40, 46 and 74 in ECW increased gradually with the development of the placenta. On the contrary, expressions of CaDna)70 were down-regulated with the development of the placenta and were scarcely expressed in B10. Furthermore, expressions of CaDnaJ10 and 56 remained nearly stable during the whole-placenta develop stage (Supplemental Figure S1).

The expressions of CaDnaJ47, 56 and 70 in CM334 were higher than those in ECW during whole development of placenta, while the CaDnaJ10 and 25 showed higher expressions only after the stage of MG. On the contrary, the expressions of CaDnaJ40 and 46 were lower in CM334 than those in ECW during whole development of placenta.

In order to verify the expressions of eight $\mathrm{CaDna}$ genes in the non-pungent and pungent cultivar for
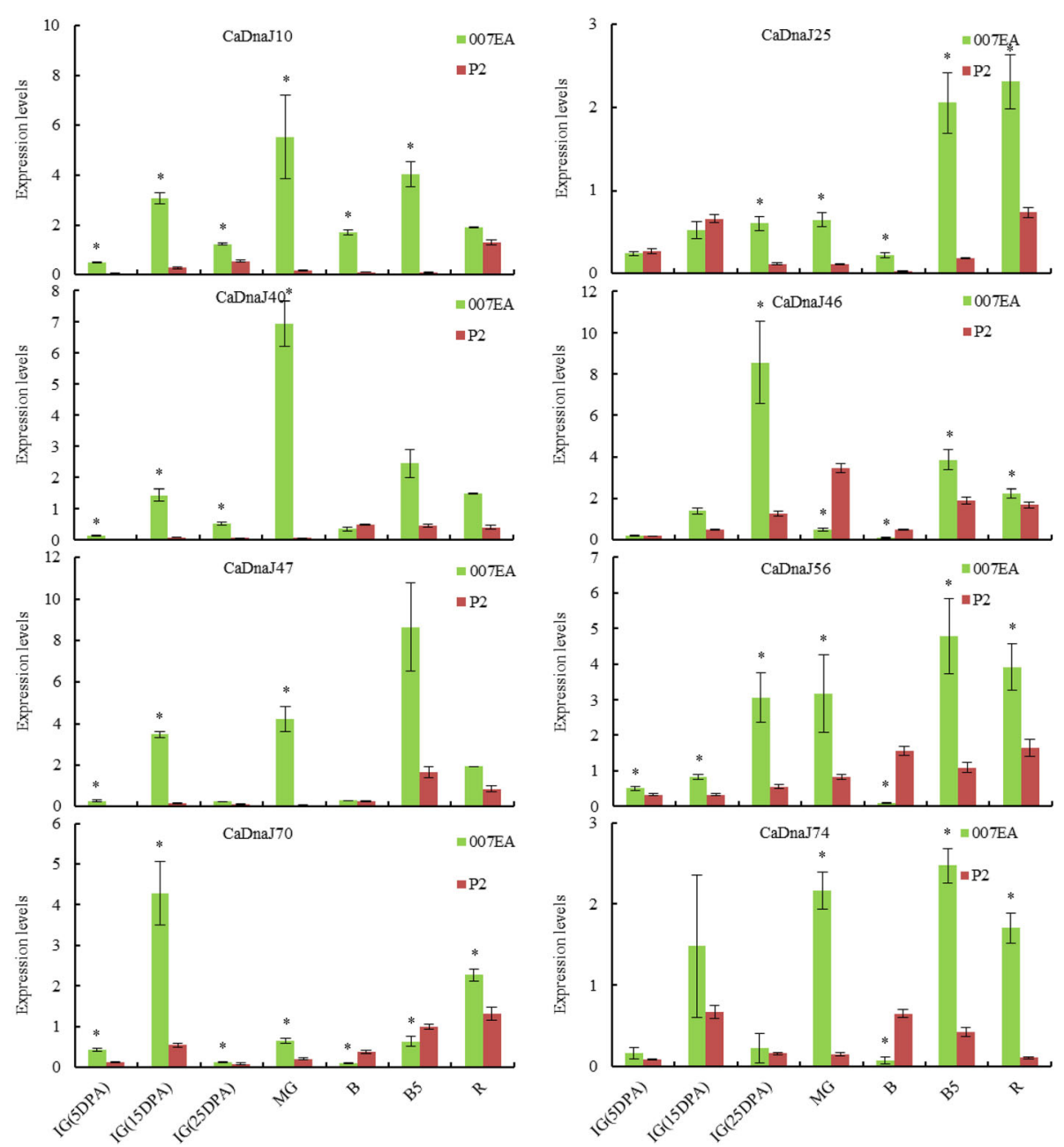

Fig. 5 The relative expressions of 8 CaDnaJ genes in placenta from 007EA (pungent) and P2 (non-pungent) on 7 different development stage. The expression levels of these CaDnaJs were tested using qRT-PCR. IG (5DPA), immature green fruit (5 days post-anthesis); IG (15DPA), immature green fruit (15 days post-anthesis); IG (25DPA), immature green fruit (25 days post-anthesis); MG, mature green fruit; B, breaker fruits; B5, breaker+ 5 fruits; R, mature red fruits. qRT-qPCR was performed in three biological replicates of different development stages of fruit samples. Bars represent the standard deviation $\left( \pm\right.$ SD) calculated for three biological replicates. Statistically significant differences are indicated $p<0.05$ by star $\left(^{*}\right)$ (Student's t-test) 
further, two accessions, the pepper P2 (non-pungent) and 007EA (pungent) at different development stages of placenta, were used to test and confirm their expressions by qRT-PCR. As shown in Fig. 5, all eight CaDnaJ genes showed distinct expression profiles. In P2 and 007EA, the expression of 6 genes (CaDnaJ10, 25, 56, 70, 47 and 74) out of $8 \mathrm{CaDnaJs}$ were mostly consistent with data from the RNA-seq in CM334 and ECW. Detail analysis revealed that the expression of CaDnaJ10, 25, 40, and 47 were up-regulated at different placenta development stages. The expression levels of CaDnaJ56, CaDnaJ70 and $\mathrm{CaDnaJ74}$ were down-regulated at the stage of $\mathrm{B}$, but were up-regulated at the other six stages. On the contrary, in P2 and 007EA, the expression of CaDnaJ40 showed opposed results from the CM334 and ECW.

\section{The CaDnaJs co-expressed with genes involved in} capsaicinoids synthesis during placenta development

The specific regulations of CaDnaJs at placenta development stages prompted us to examine whether the CaDnaJs co-expressed with genes involved in capsaicinoids synthesis. Previously, nine capsaicinoidbiosynthetic genes (CBGs) have been identified [16]. In this study, expression patterns of these nine genes during placenta development were performed based RNA-seq (Supplemental Figure S2). The result showed that expression levels of all genes related to capsaicinoids synthesis in immature green fruit (5DPA, 15DPA and 25DPA) were higher than that in mature red fruit (break, break+ 5 and red fruit). Among these nine genes, expressions of three genes (Pal1, BCAT and $\mathrm{C} 4 \mathrm{H}$ ) in 007EA (high pungent) were lower than that in P2 (non-pungent), which does not relate to the capsaicin accumulation at placenta developmental stage. For KAS and FatA genes, we found that expression levels of these two genes were higher in P2 than that in 007EA at 15DPA; however, they were lower in fruit at 25DPA. These two genes (KAS and FatA) were potentially co-expressed with $\mathrm{CaDnaJs}$ at immature fruit (25 DPA) (Supplemental Figure S1). Expression of the remaining four genes (ACL, COMT, AMT and CS) showed higher in 007EA than that in P2, which was consistent with expression of CaDnaJs (Supplemental Figure S1). Overall, these nine genes in the pepper genome by examining their tissue-specific expression profiles indicated that six genes appear to be coexpressed with CaDnaJs during placenta development.

\section{Expression patterns of CaDnaJ genes in response to different kinds of stress condition}

Abiotic stresses like salinity, heat, and polyethylene glycol (PEG) adversely affect the growth and physiological processes of plants [28-39]. In this study, expression
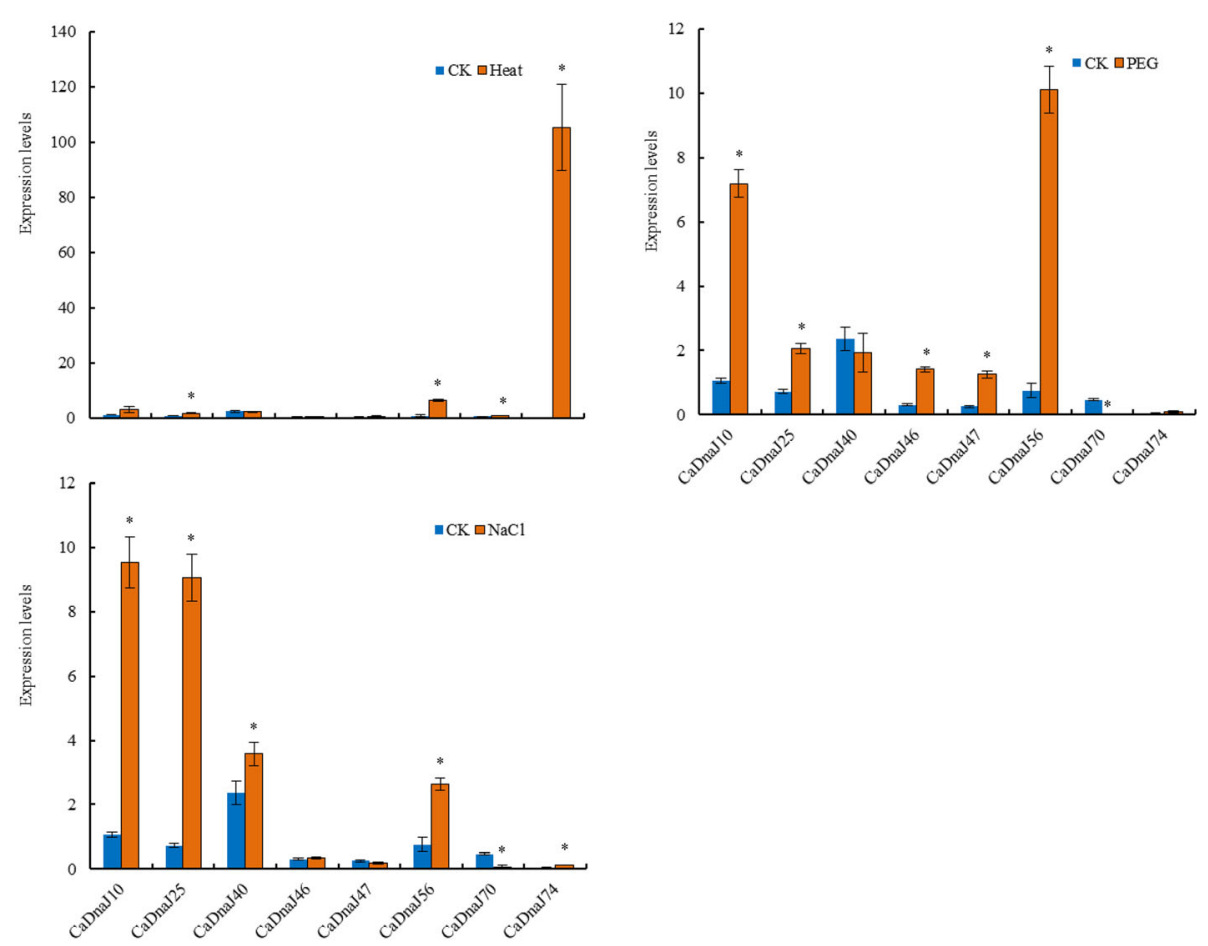

Fig. 6 The relative expressions of 8 CaDnaJ genes in the leaves of 007EA (pungent) at the stage of 6-8 true leaves in response to abiotic stresses, including heat, PEG and Nacl. Each treatment was conducted with three biological replicates, and samples from five plants were collected for each replicate. qRT-qPCR was performed in three biological replicates of the samples. Bars represent the standard deviation ( \pm SD) calculated for three biological replicates. Statistically significant differences are indicated $p<0.05$ by star ${ }^{*}$ ) (Student's t-test) 
patterns of eight CaDnaJ genes related to biosynthesis of compounds related with pungency were further investigated under various abiotic stresses to explore their potential roles in stress tolerance. With the help of qPCR, the response of $8 \mathrm{CaDnaJs}$ to stress conditions, including heat, PEG and salt, was analyzed. The results showed that these eight genes were regulated by heat, PEG and salt, respectively (Fig. 6). The expression of CaDnaJ74 was greatly up-regulated under high temperature stress, implying that CaDnaJ74 may be involved in the process of plant response to high-temperature stress. Under drought stress, the expression of eight $\mathrm{CaDnaJ}$ genes presented noticeable changes. This result showed that expressions of 5 genes (CaDna)10, 25, 46, 47 and 56) out of $8 \mathrm{CaD}$ naJs were significantly up-regulated. By contrast, $\mathrm{CaD}$ naJ70 was down-regulated. The results imply that these CaDnaJs might participate in drought stress responses. Compared to expression levels under drought stress, only $\mathrm{CaDna} 70$ and 74 were down-regulated during salt stress.

\section{Expression patterns of CaDnaJ genes under hormone treatment}

Plant hormones regulate multiple aspects of plant growth and development and mediate environmental responses to ensure a successful life cycle [27, 40-47]. In this study, in order to investigate the roles of the CaDnaJ genes in response to hormone treatments, the seedlings at the age of 6-8 true leaves were subjected to abscisic acid (ABA), $\mathrm{GA}_{3}$, methyl jasmonate (MeJA) and salicylic acid (SA) treatments (Fig. 7). For ABA treatment, four genes (CaDnaJ10, 25, 40 and 46) were remarkably upregulated (Fig. 7). For $\mathrm{GA}_{3}$ treatment, CaDnaJ10, 25, 40, 46,47 and 74 also were up-regulation except for CaDnaJ56 and 70 genes. Both MeJA and SA treatments induced up-regulation of 7 genes (CaDnaJ10, 25, 40, 46, 47, 56 and 74). On the contrary, CaDnaJ70 was downregulated in all tested hormone treatments (Fig. 7). Our results suggested that these eight $\mathrm{CaDnaJ}$ genes were induced by these hormone treatments.

\section{Discussion}

Because of its rich capsaicin, nutrients and pigments in the fruits, pepper has become the world's second most popular vegetable after tomato [48, 49]. Recently, the DnaJ proteins, ubiquitously participate in various plant growth and development processes, have been identified in pepper [23]. In the present study, the comprehensive expression profiles of entire 76 pepper DnaJ genes were analyzed in detail. Our result showed that about 38\% (29) of CaDnaJs were expressed in at least one tissues
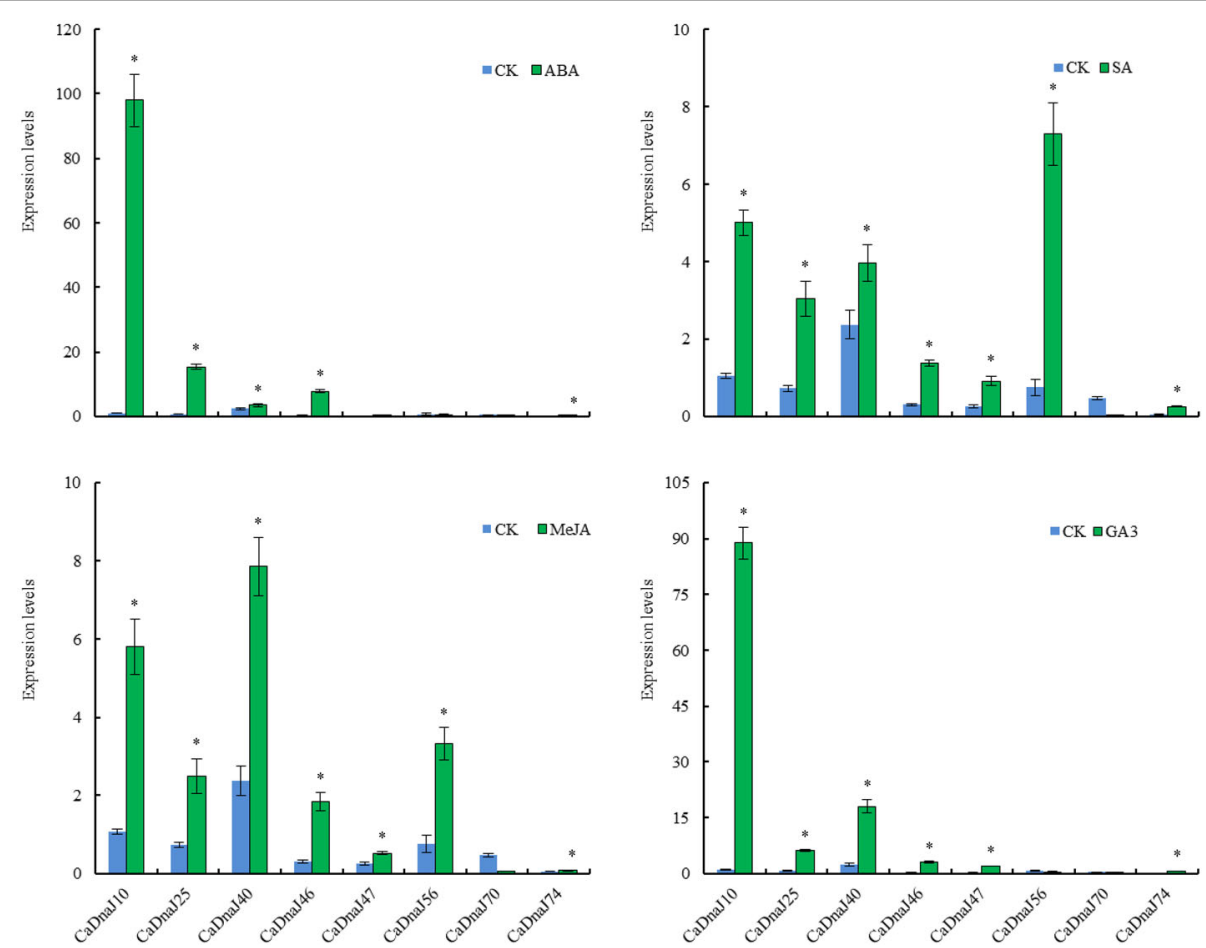

Fig. 7 The relative expressions of 8 CaDnaJ genes in the leaves of 007EA (pungent) at the stage of 6-8 true leaves in response to plant hormones, including ABA, GA3, MeJA and SA. Each treatment was conducted with three biological replicates, and samples from five plants were collected for each replicate. qRT-qPCR was performed in three biological replicates of of the samples. Bars represent the standard deviation ( \pm SD) calculated for three biological replicates. Statistically significant differences are indicated $p<0.05$ by star (*) (Student's t-test) 
(Supplement Table S2), indicating the potentially critical role of CaDnaJs in plant growth and development in pepper. Further analysis of these $29 \mathrm{CaDnaJs}$ revealed that 5, 6 and 3 CaDnaJs were highly expresssed in leaf, root and stem, respectively, suggesting fundamental function in pepper vegetative growth stage (Supplement Table S2; Table 2). Additionally, we also found that 10 and $5 \mathrm{CaDnaJs}$ were highly expresssed in pericarp and placenta, respectively, indicating a conservation role of these genes in pepper reproductive growth stage (Supplement Table S2; Table 2). Moreover, expression of some CaDnaJ genes was tissue-specific. For example, CaDnaJ27 and 66 were specifically expressed in leaf. Seven genes, CaDnaJ 07, 10, 13, 44, 45, 46 and 74, were specifically expressed in root. The CaDnaJ 04 was specifically expressed in pericarp. These results revealed that these genes may be associated with the regulation of growth and development of these specific tissues.

The pungent properties of peppers are attributed to a group of compounds called capsaicinoids, unique alkaloids that accumulate only in pepper fruit. These compounds are becoming increasingly important in food, cosmetic and pharmaceutical industries. The capsaicinoids are found generally in the white part inside the pepper, which were called placenta. Currently, the capsaicinoids biosynthetic pathways, including the key CBGs and the related transcription factors (TFs), such as Erf, Jerf and CaMYB31, have been largely elucidated [50, 51]. To analyze the relationship between expression profiles of DnaJ genes and capsaicinoids synthesis, nine genes involved in capsaicinoids synthesis (Pal1, BCAT, C4H, KAS, ACL, COMT, FatA, AMT and CS) were selected in the pungent (007EA) and non-pungent peppers (P2) in this study [16]. Among them, we found that six genes (KAS, FatA, ACL, COMT, AMT and CS) genes were higher in P2 than that in 007EA at IG (5DPA, 15DPA and 25DPA), which was consistent with expressions of eight CaDnaJs during placenta development. Co-expression patterns between CaDnaJs and the genes related to capsaicinoids synthesis were found, which implied that these CaDnaJs genes might participate in the regulation of capsaicinoids synthesis in pepper.

Usually, understanding of how and where each gene functions demands detailed knowledge of spatialtemporal gene expression patterns $[43,52,53]$ and in response to various abiotic stresses [54-57]. The biosynthesis and accumulation of capsaicinoids in chili pepper fruits were affected by various internal and environmental factors [58], including plant hormones like indoleacetic acid (IAA), jasmonic acid (JA), salicylic acid (SA), and gibberellic acid (GA), and stress conditions such as temperature, light, wounding and drought. Moreover, the environment effects on fruit yield and capsaicinoids production might vary depending on the chili genotypes.
Previous studies in seven hot pepper hybrid cultivars and two commercial cultivars confirmed that a large proportion of variation on capsaicinoid yield (67.7\%) was contributed from environment condition while variations due to genotype were only $42.4 \%$ [50,59]. On the contrary, Tripodi et al. [60] reported that the environment accounted for less than $0.5 \%$ for capsaicinoids in 14 hot pepper accessions cultivated in two different pedoclimatic locations.

In our study, eight CaDnaJ genes (CaDna)10, 25, 40, $46,47,56,70$ and 74 ) with similar expression pattern to the CBGs in placenta of CM334 (pungent) and ECW (non-pungent) were selected to investigate their potential roles in pepper responses to various abiotic stresses and hormones. The expression levels of these eight CaDnaJ genes under heat, drought and salt were determined by qRT-PCR (Fig. 6). Some researchers had suggested that capsaicin production was improved under limited water supply in the low and medium pungency cultivars [61]. In contrast, the reduction or no changes of capsaicinoid production under drought condition also was found $[59,62]$. Our data showed that drought condition had greater effect on the expression of these eight genes than the other two abiotic stresses. The expressions of 6 genes out of $8 \mathrm{CaDnaJs}$ were significantly increased in response to drought stress. The expression levels of only two genes (CaDnaJ40 and CaDnaJ70) were slightly decreased. The result supported previous study that the synthesis of capsaicinoids was sensitive to water stress.

In previous studies, it was reported that SA, IAA and $\mathrm{GA}_{3}$ treatment promoted the expression of three capsaicinoid structural genes, CaMYB31, Kas, and pAmt, while JA treatment caused a significant decrease in their expression, except at $3 \mathrm{~h}$ of exposure [50]. In our study, the qRT-PCR analysis showed that the expression of these eight CaDanJ genes was up-regulated or downregulated after 4 different hormone treatments including $\mathrm{ABA}, \mathrm{GA}_{3}, \mathrm{MeJA}$ and SA (Fig. 7). However, we did not observe an antagonistic effect of MeJA on the expression of these eight CaDanJ genes compared with the $\mathrm{ABA}$, $\mathrm{GA}_{3}$ and SA. This phenomenon supported the fact that the level of pungency in hot pepper was either positively or negatively regulated by different plant hormones. The concentration of the plant hormone and exposure time highly influence the pungency level.

\section{Conclusions}

In our study, a genome-wide identification and expression analysis of the $76 \mathrm{CaDnaJs}$ were performed at the whole genome level. Eight DnaJ genes involved in the regulation of capsaicinoids synthesis were confirmed by the co-expression patterns of these $\mathrm{CaDnaJ}$ genes and CBGs. Furthermore, these eight genes were induced by 
various abiotic stresses (heat, drought and salt) and plant hormones (ABA, GA3, MeJA and SA), which revealed the influence of environmental factors in the biosynthesis of compounds related with pungency. These results provide new information that may facilitate the further functional analysis of the pepper CaDnaJs.

\section{Methods}

\section{Data sources}

The genomic sequences of pepper downloaded from the Pepper Genome Database (PGD, http://peppergenome. snu.ac.kr/) [16].

\section{Phylogenetics analysis}

The full amino acid sequences of $76 \mathrm{CaDnaJ}$ members from Capsicum annuum CM334 were aligned by ClustalX program. The phylogenetic tree was built using MEGA5.10 software [63]. The Neighbor-joining method, pair wise deletion and a Poisson model were used with a bootstrap (1000 replicates) test of phylogeny. The pepper $\mathrm{CaDnaJ}$ genes were assigned to different groups based on the multiple sequence alignment and the classification of DnaJ genes in the other four species.

\section{Expression analysis of pepper DnaJ in various tissues}

RNA-seq data, which have been revealed by previous researchers [16], were used to investigate expression patterns of CaDnaJ genes in the roots, stems, and leaves from 6-week-old CM334 plants.

RPKM (Reads Per Kilo bases per Million mapped Reads) values of CaDnaJ genes were log2- transformed [64]. Based on the expression levels and patterns, the genes were defined as expressed, specifically expressed and preferentially expressed according to the classification criteria of Cheng et al. [65].

\section{Plant growth and stress treatments}

Two inbred lines provided by our lab, P2 (non-pungent) and 007EA (pungent), were cultivated in the greenhouse at the Zhejiang Academy of Agricultural Sciences. The placenta were harvested from P2 and 007EA plants to investigate expression patterns of CaDnaJ genes at different development stage, including immature green fruit (5DPA, 15DPA and 25DPA), mature green fruit, breaker fruits, breaker+ 5 fruits and mature red fruits.

For hormone treatments, seeds for hot pepper 007EA were sterilized for $5 \mathrm{~min}$ using $10 \%$ hypochlorous acid solution. Germinated seeds were sown in plastic pots with substrate (peat:perlite-3:1 by volume) in a growth chamber, under $16-\mathrm{h}$ photoperiod at $26^{\circ} \mathrm{C} / 19^{\circ} \mathrm{C}$ till reaching the age of 6-8 true leaves. Subsequently, the seedlings were sprayed with $100 \mu \mathrm{M}$ Methyl jasmonate (MeJA), $100 \mu \mathrm{M}$ Gibberellin $\left(\mathrm{GA}_{3}\right), 100 \mu \mathrm{M}$ abscisic acid
(ABA), $1000 \mu \mathrm{M}$ salicylic acid (SA) solutions, and leaves were collected $4 \mathrm{~h}$ later.

The seedlings of hot pepper var. 007EA were root irrigated $200 \mathrm{~mL}$ of $50 \mathrm{mM} \mathrm{NaCl}$ solution to the substrate per plant for salt stress, and $200 \mathrm{~mL}$ of $18 \%$ PEG for drought stress treatments. For heat stress treatments, the plants were treated with $42^{\circ} \mathrm{C}$ by placing in a light incubator and plants grown at $25^{\circ} \mathrm{C}$ were used as the control group. The leaves of all treatments were sampled for analysis $4 \mathrm{~h}$ later.

Three biological replicates of samples of hormone treatments (MeJA, $\mathrm{GA}_{3}, \mathrm{ABA}$ and $\mathrm{SA}$ ) and abiotic stress treatments $\left(42^{\circ} \mathrm{C}\right.$ and $\left.\mathrm{Nacl}\right)$ were collected from pepper 007EA planted in the greenhouse of Zhejiang Academy of Agricultural Sciences (Hangzhou, China). Each biological replicate contains five individuals. We collected five leaves from each individual and mixed them together as one biological replicate. The samples were collected and immediately frozen with liquid nitrogen for total RNA extraction.

\section{Total RNA extraction, reverse transcriptions and qRT-PCR analysis}

Total RNA extraction and reverse transcription were performed using Total RNA kit and FastQuant RT Kit (Tiangen Biotech, Beijing, China) according to the manufacturers' protocol.

The ABI StepOne Plus Real-time PCR system was used for quantitative RT-PCR analysis. The procedure was done according to the instructions in the SYBR qPCR Master Mix (Vazyme Biotech co., Ltd., Nanjing, China). Cycling conditions were $94^{\circ} \mathrm{C}$ for $5 \mathrm{~min}$, followed by 33 cycles of $94{ }^{\circ} \mathrm{C}$ for $30 \mathrm{~s}, 55^{\circ} \mathrm{C}$ for $30 \mathrm{~s}$ and extension at $72{ }^{\circ} \mathrm{C}$ for 30 s. The $U B I$ gene was used as an internal control [66]. Gene-specific primers used for amplification were listed in Supplement Table S1. The expression values of $\mathrm{CaDnaJ}$ genes were calculated by the $2^{-\Delta \mathrm{ct}}$ method.

\section{Statistical analysis}

All experiments were done with three biological repeats, and five plants per treatment were collected for analysis. All data were statistically analyzed with SPSS software (https://www.ibm.com/analytics/spss-statistics-software) using student's t-test at the 0.05 level of significance.

\section{Supplementary information}

Supplementary information accompanies this paper at https://doi.org/10. 1186/s12870-020-02476-3.

Additional file 1 Table $\mathbf{S 1}$ Primers used for $\mathrm{qRT}$-PCR in this study. Table S2 Fragments per kilobase of exon model per million mapped (FPKM) values of all the CaDnaJs in different pepper tissues. Supplemental Fig. S1 The relative expressions of 8 CaDnaJ genes in placenta from CM334 (pungent) and ECW (non-pungent) based on RNA-seq data. 6DPA, 
6 days post-anthesis; 16 DPA, 16 days post-anthesis; 25 DPA, 25 days postanthesis; MG, mature green; $B 5,5$ days post-breaker; $B 10,10$ days postbreaker. Supplemental Fig. S2 The RNA-Seq data of nine capsaicinoidbiosynthetic genes during the different stage of placenta from CM334 (pungent) and ECW (non-pungent). IG (6DPA), immature green fruit (5 days post-anthesis); IG (16DPA), immature green fruit (16 days postanthesis); IG (25DPA), immature green fruit (25 days post-anthesis); $M G$, mature green fruit; $B$, breaker fruits; $B 5$, breaker+ 5 fruits; $R$, mature red fruits.

\section{Abbreviations}

ABA: Abscisic acid; B: Breaker; B5: 5 days post-breaker; CBGs: Capsaicinoidbiosynthetic genes; DPA: Days post-anthesis; GA3: Gibberellin; IAA: Indoleacetic acid; JA: Jasmonic acid; MeJA: Methyl jasmonate; MG: Mature green; RPKM: Reads Per Kilo bases per Million mapped Reads: SA: Salicylic acid; TFs: Transcription factors

\section{Acknowledgements}

Not applicable.

\section{Authors' contributions}

FF, FL performed and analyzed most of the experiments in this study, with assistance from XY provided all financial support and critical intellectual input in the design of this study and preparation of the manuscript. HW and YK designed this study and wrote the manuscript. All authors discussed the results and commented on the manuscript. The authors read and approved the final manuscript.

\section{Funding}

This research was partially supported by the Guangdong Provincial Special Fund For Modern Agriculture Industry Technology Innovation Teams (No. 2019KJ122), Zhejiang Provincial Natural Science Foundation of China (LY18C150008), National Natural Science Foundation of China (31872090), National Key Research and Development Program (2017YFD0101902), Modern Agricultural Industry Technology system construction of Guangdong, China, State Key Laboratory Breeding Base for Zhejiang Sustainable Pest and Disease Control (No. 2010DS700124-ZZ1807), the Earmarked Fund for China Agriculture Research System (CARS-23-G44), Zhejiang Provincial major Agricultural Science and Technology Projects of New Varieties Breeding (2016C02051). The funders had no role in the design of the study and collection, analysis, and interpretation of data and in writing the manuscript.

\section{Availability of data and materials}

All data used during the current study are included in this published article or are available from the corresponding author on reasonable request.

\section{Ethics approval and consent to participate}

Not applicable.

\section{Consent for publication}

Not applicable.

\section{Competing interests}

The authors declare that they have no competing interests.

\section{Author details}

${ }^{1}$ College of Horticulture, South China Agricultural University, Guangzhou 510642, PR China. ${ }^{2}$ Horticultural Research Institute, Yunnan Academy of Agricultural Science, Kunming 650231, PR China. ${ }^{3}$ State Key Laboratory for Managing Biotic and Chemical Threats to the Quality and Safety of Agro-products, Institute of Vegetables, Zhejiang Academy of Agricultural Sciences, Hangzhou 310021, PR China. ${ }^{4}$ China-Australia Research Centre for Crop Improvement, Zhejiang Academy of Agricultural Sciences, Hangzhou 310021, China.
Received: 9 June 2019 Accepted: 1 June 2020

Published online: 09 July 2020

\section{References}

1. Georgopoulos CP, Lundquist-Heil A, Yochem J, Feiss M. Identification of the E. coli DnaJ gene product. Mol Gen Genet. 1980;178:583-8.

2. Bukau B, Horwich AL. The Hsp70 and Hsp60 chaperone machines. Cell. 1998:92:351-66.

3. Caplan AJ, Cyr DM, Douglas MG. Eukaryotic homologues of Escherichia coli DnaJ: a diverse protein family that functions with Hsp70 stress proteins. Mol Biol Cell. 1993:4:555-63.

4. Craig EA, Huang P, Aron R, Andrew A. The diverse roles of J-proteins, the obligate Hsp70 co-chaperone. Rev Physiol Biochem Pharmacol. 2006;156:121.

5. Silver PA, Way JC. Eukaryotic DnaJ homologs and the specificity of Hsp70 activity. Cell. 1993;74:5-6

6. Cheetham ME, Caplan AJ. Structure, function and evolution of DnaJ: conservation and adaptation of chaperone function. Cell Stress Chaperones. 1998;3(1):28-36.

7. Ohtsuka K, Hata M. Mammalian HSP40/DNAJ homologs: cloning of novel cDNAs and a proposal for their classification and nomenclature. Cell Stress Chaperones. 2000:5:98-112.

8. Xing Y, Zhang Q. Genetic and molecular bases of rice yield. Annu Rev Plant Biol. 2010:61:421-42.

9. Vitha S, Froehlich JE, Koksharova O, Pyke KA, van Erp H, Osteryoung KW. ARC6 is a J-domain plastid division protein and an evolutionary descendant of the cyanobacterial cell division protein Ftn2. Plant Cell. 2003;15:1918-33.

10. Chen KM, Holmström M, Raksajit W, Suorsa M, Piippo M, Aro EM. Small chloroplast-targeted DnaJ proteins are involved in optimization of photosynthetic reactions in Arabidopsis thaliana. BMC Plant Biol. 2010;10(1): 43.

11. Wang YW, Chen SM, Wang WJ, Huang XQ, Zhou CF, Zhuang Z, et al. The DnaJ-like zinc finger domain protein PSA2 affects light acclimation and chloroplast development in Arabidopsis thaliana. Front Plant Sci. 2016;7:360.

12. Zhu X, Liang S, Yin J, Yuan C, Wang J, Li W, et al. The DnaJ OsDjA7/8 is essential for chloroplast development in rice (Oryza sativa). Gene. 2015; 574(1):11-9.

13. Rajan VB, D'Silva P. Arabidopsis thaliana J-class heat shock proteins: cellular stress sensors. Funct Integr Genomics. 2009;9:433-46.

14. Bekh-Ochir D, Shimada S, Yamagami A, Kanda S, Ogawa K, Nakazawa M, et al. A novel mitochondrial DnaJ/Hsp40 family protein BIL2 promotes plant growth and resistance against environmental stress in brassinosteroid signaling. Planta. 2013;237(6):1509-25.

15. Kong F, Deng Y, Zhou B, Wang G, Wang Y, Meng Q. A chloroplast-targeted DnaJ protein contributes to maintenance of photosystem II under chilling stress. J Exp Bot. 2014;65(1):143-58.

16. Kim S, Park M, Yeom SI, Kim YM, Lee JM, Lee HA, et al. Genome sequence of the hot pepper provides insights into the evolution of pungency in Capsicum species. Nat Genet. 2014;46:270-8.

17. Bennett DJ, Kirby GW. Constitution and biosynthesis of capsaicin. J Chem Soc C. 1968:442-6.

18. Aza-González C, Núñez-Palenius HG, Ochoa-Alejo N. Molecular biology of capsaicinoid biosynthesis in Chili pepper (Capsicum Spp.). Plant Cell Rep. 2011;30(5):695-706.

19. Chen MY, Li K, Li HP, Song CP, Miao YC. The glutathione peroxidase gene family in Gossypium hirsutum: genome-wide identification, classification, gene expression and functional analysis. Sci Rep. 2017;7:44743.

20. Guo YW, Guo HL, Li X, Huang LL, Zhang BN, Pang XB, et al. Two type III polyketide synthases from Polygonum cuspidatum: gene structure, evolutionary route and metabolites. Plant Biotech Rep. 2013;7(3):371-81.

21. Lu T, Zhang G, Sun L, Wang J, Hao F. Genome-wide identification of CBL family and expression analysis of CBLs in response to potassium deficiency in cotton. Peer J. 2017;5:e3653.

22. Sun Q, Wang GH, Zhang X, Zhang XR, Qiao P, Long L, et al. Genome-wide identification of the TIFY gene family in three cultivated Gossypium species and the expression of JAZ genes. Sci Rep. 2017;7:42418.

23. Fan F, Yang $X$, Cheng $Y$, Kang $Y$, Chai $X$. The DnaJ gene family in pepper (Capsicum annuum L.): comprehensive identification, characterization and expression profiles. Front. Plant Sci. 2017;8:689.

24. Han YJ, Wang XH, Chen WC, Dong MF, Yuan WJ, Liu X, et al. Differentia expression of carotenoid-related genes determines diversified carotenoid 
coloration in flower petal of Osmanthus fragrans, Tree Genet. Genomes 2014;10(2):329-38

25. Han Y, Wu M, Cao L, Yuan W, Dong M, Wang X, et al. Characterization of OfWRKY3, a transcription factor that positively regulates the carotenoid cleavage dioxygenase gene OfCCD4 in Osmanthus fragrans. Plant Mol Biol. 2016;91(4-5):485-96

26. Liang JY, Xia JY, Liu LL, Wan SQ. Global patterns of the responses of leaflevel photosynthesis and respiration in terrestrial plants to experimental warming. J Plant Ecol. 2013;6(6):437-47.

27. Zhao X, Wang YL, Qiao XR, Wang J, Wang LD, Xu CS, et al. Phototropins function in high-intensity blue light-induced hypocotyl phototropism in Arabidopsis by altering cytosolic calcium. Plant Physiol. 2013;162(3):1539-51.

28. Gao W, Xu FC, Guo DD, Zhao JR, Liu J, Guo YW, et al. Calcium-dependent protein kinases in cotton: insights into early plant responses to salt stress. BMC Plant Biol. 2018;18(1):15.

29. Li W, Zhao F, Fang W, Xi ED, Hou J, Yang X, et al. Identification of early salt stress responsive proteins in seedling roots of upland cotton (Gossypium hirsutum L.) employing iTRAQ-based proteomic technique. Front Plant Sci. 2015;11(6):732.

30. Lü D, Wang W, Miao C. ATHK1 acts downstream of hydrogen peroxide to mediate ABA signaling through regulation of calcium channel activity in Arabidopsis guard cells. Chin Sci Bull. 2013;58(3):336-43.

31. Ma XN, Zhang XR, Yang L, Tang MM, Wang K, Wang L, et al. Hydrogen peroxide plays an important role in PERK4-mediated abscisic acid-regulated root growth in Arabidopsis. Funct Plant Biol. 2018;46(2):165-74.

32. Qi J, Song CP, Wang B, Zhou J, Kangasjärvi J, Zhu JK, et al. Reactive oxygen species signaling and stomatal movement in plant responses to drought stress and pathogen attack. J Integr Plant Biol. 2018;60(9):805-26.

33. Wang PT, Liu H, Hua HJ, Wang L, Song CP. A vacuole localized betaglucosidase contributes to drought tolerance in Arabidopsis. Chin Sci Bull. 2011;2011(56):3538-46.

34. Xu LH, Wang WY, Guo JJ, Qin J, Shi DQ, Li YL, et al. Zinc improves salt tolerance by increasing reactive oxygen species scavenging and reducing $\mathrm{Na}+$ accumulation in wheat seedlings. Biol Plantarum. 2014; 58(4):751-7.

35. Zhang J, Wang F, Zhang C, Zhang J, Chen Y, Liu G, et al. A novel VIGS method by agroinoculation of cotton seeds and application for elucidating functions of GhBl-1 in salt-stress response. Plant Cell Rep. 2018;37(8):1091-100.

36. Zhao Q, Chen W, Bian J, Xie H, Li Y, Xu C, et al. Proteomics and phosphoproteomics of heat stress-responsive mechanisms in spinach. Front Plant Sci. 2018:9:800

37. Ma $L$, Zhang $H$, Sun $L$, Jiao $Y$, Zhang $G$, Miao $C$, et al. NADPH oxidase AtrbohD and AtrbohF function in ROS-dependent regulation of $\mathrm{Na}^{+} / \mathrm{K}^{+}$ homeostasis in Arabidopsis under salt stress. J Exp Bot. 2012;63(1):305-17.

38. Zhao $X$, Wang $Y$ J, Wang $Y L$, Wang $X L$, Zhang $X$. Extracellular $\mathrm{Ca}^{2+}$ alleviates $\mathrm{NaCl}$-induced stomatal opening through a pathway involving $\mathrm{H}_{2} \mathrm{O}_{2}$-blocked $\mathrm{Na}^{+}$influx in Vicia guard cells. J Plant Physiol. 2011;168(9):903-10.

39. Zhao X, Li YY, Xiao HL, Xu CS, Zhang X. Nitric oxide blocks blue lightinduced $\mathrm{K}^{(+)}$influx by elevating the cytosolic $\mathrm{Ca}^{2+}$ concentration in Vicia faba L guard cells. J Integr Plant Biol. 2013;55(6):527-36.

40. Hao F, Zhao S, Dong H, Zhang H, Sun L, Miao C. Nia1 and Nia2 are involved in exogenous salicylic acid-induced nitric oxide generation and stomatal closure in Arabidopsis. J Integr Plant Biol. 2010;52(3):298-307.

41. Li K, Yang FB, Miao YC, Song CP. Abscisic acid signaling is involved in regulating the mitogen-activated protein kinase cascade module, AIK1MKK5-MPK6. BMC Plant Biol. 2017;12:5.

42. Li L, Hou MJ, Cao L, Xia Y, Shen ZG, Hua ZB. Glutathione S-transferases modulate cu tolerance in Oryza sativa. Environ Exp Bot. 2018a;155:313-20.

43. Li W, de Ollas C, Dodd IC. Long-distance ABA transport can mediate distal tissue responses by affecting local ABA concentrations. J Integr Plant Biol. 2018b;60(1):16-33.

44. Liu LY, Li N, Yao CP, Meng SS, Song CP. Functional analysis of the ABAresponsive protein family in $A B A$ and stress signal transduction in Arabidopsis. Chin Sci Bull. 2013;58:3721-30.

45. Song Y, Xiang F, Zhang G, Miao Y, Miao C, Song CP. Abscisic acid as an internal integrator of multiple physiological processes modulates leaf senescence onset in Arabidopsis thaliana. Front Plant Sci. 2016;19(7):181.

46. Wang $K$, He JN, Zhao Y, Wu T, Zhou XF, Ding YL, et al. EAR1 negatively regulates $A B A$ signaling by enhancing $2 C$ protein phosphatase activity. Plant Cell. 2018;30:815-34.
47. Zhang G, Lu T, Miao W, Sun L, Tian M, Wang J, et al. Genome-wide identification of ABA receptor PYL family and expression analysis of PYLs in response to ABA and osmotic stress in Gossypium. Peer J. 2017;5:e4126.

48. Benson GAS, Obadofin AA, Adesina JM. Evaluation of plant extracts for controlling insect pests of pepper (Caspicum spp.) in Nigeria humid rainforest. New York Sci J. 2014;7(1):39-43.

49. Tian SL, Lu BY, Gong ZH, Muhammad-Shah SN. Effects of drought stress on capsanthin during fruit development and ripening in pepper (Capsicum annuum L.). Agric Water Manag. 2014;137:46-51.

50. Arce-Rodríguez ML, Ochoa-Alejo N. An R2R3-MYB transcription factor regulates capsaicinoid biosynthesis. Plant Physiol. 2017;174:1359-70.

51. Zhu ZS, Sun BM, Cai W, Zhou X, Mao YH, Chen CJ, et al. Natural variations in the MYB transcription factor MYB31 determine the evolution of extremely pungent peppers. New Phytol. 2019;223(2):922-38.

52. Pang YQ, Li JT, Qi BS, Tian M, Sun LR, Wang XC, et al. Aquaporin AtTIP5;1 as an essential target of gibberellins promotes hypocotyl cell elongation in Arabidopsis thaliana under excess boron stress. Funct Plant Biol. 2017;45(3): 305-14.

53. Sun $L R$, Ma $L Y, H e S B$, Hao F, H. AtrbohD functions downstream of ROP2 and positively regulates waterlogging response in Arabidopsis. Plant Signal Behav. 2018;13(9):1-5.

54. Guo S, Dai S, Singh PK, Wang H, Wang Y, Tan JLH, et al. A membranebound NAC-like transcription factor OsNTL5 represses the flowering in Oryza sativa. Front Plant Sci. 2018;9:555.

55. Lv SF, Yu DY, Sun QQ, Jiang J. Activation of gibberellin 20-oxidase 2 undermines auxin-dependent root and root hair growth in $\mathrm{NaCl}$-stressed Arabidopsis seedlings. Plant Growth Regul. 2018;84:225-36.

56. Shang BS, Zang YH, Zhao X, Zhu JD, Fan C, Guo XN, et al. Functional characterization of GhPHOT2 in chloroplast avoidance of Gossypium hirsutum. Plant Physiol Biochem. 2019;135:51-60.

57. Xu FC, Liu HL, Xu YY, Zhao JR, Guo YW, Long L, et al. Heterogeneous expression of the cotton R2R3-MYB transcription factor GbMYB60 increases salt sensitivity in transgenic Arabidopsis. Plant Cell Tiss Org Cult. 2018;133: 15-25.

58. Gurung T, Techawongstien S, Suriharn B, Techawongstien S. Impact of environments on the accumulation of capsaicinoids in Capsicum spp. HortSci. 2011;46:1576-81.

59. Jeeatid N, Suriharn B, Techawongstien S, Chanthai S, Bosland PW, Techawongstien S. Evaluation of the effect of genotype-by-environment interaction on capsaicinoid production in hot pepper hybrids (Capsicum chinense Jacq.) under controlled environment. Sci Hortic. 2018;235:334-9.

60. Tripodi P, Cardi T, Bianchi G, Migliori CA, Schiavi M, Rotino GL, et al. Genetic and environmental factors underlying variation in yield performance and bioactive compound content of hot pepper varieties (Capsicum annuum) cultivated in two contrasting Italian locations. Eur Food Res Technol. 2018; 244:1555-67.

61. Phimchan P, Chanthai S, Bosland PW, Techawongstien S. Enzymatic changes in phenylalanine ammonia-lyase, cinnamic-4-hydroxylase, capsaicin synthase, and peroxidase activities in Capsicum under drought stress. J Agric Food Chem. 2014;62:7057-62.

62. Khamsuk O, Sonjaroon W, Suwanwong S, Jutamanee K, Suksamrarn A. Effects of 24-epibrassinolide and the synthetic brassinosteroid mimic on chili pepper under drought. Acta Physiol Plant. 2018;40:106.

63. Tamura K, Peterson D, Peterson N, Stecher G, Nei M, Kumar S. MEGA5: molecular evolutionary genetics analysis using maximum likelihood, evolutionary distance, and maximum parsimony methods. Mol Biol Evol. 2011;28:2731-9.

64. Wei KF, Chen J, Chen YF, Wu LJ, Xie DX. Molecular phylogenetic and expression analysis of the complete WRKY transcription factor family in maize. DNA Res. 2012;19:153-64.

65. Cheng Y, Ahammed GJ, Yu JH, Yao ZP, Ruan MY, Ye QJ, et al. Putative WRKYs associated with regulation of fruit ripening revealed by detailed expression analysis of the WRKY gene family in pepper. Sci Rep. 2016;6:39000.

66. Wan HJ, Yuan W, Ruan MY, Ye QJ, Wang RQ, Li ZM, et al. Identification of reference genes for reverse transcription quantitative real-time $P C R$ normalization in pepper (Capsicum annuum L.). Biochem Biophys Res Commun. 2011;416:24-30.

\section{Publisher's Note}

Springer Nature remains neutral with regard to jurisdictional claims in published maps and institutional affiliations. 